Revue trimestrielle sur l'image géographique et les formes du territoire

129 | 2020

Varia

\title{
SYLVAIN SOUCHAUD, Le Brésil
}

Atlande, coll. « Clefs-concours »

\section{Nicolas Bautès}

\section{(2) OpenEdition}

Journals

Édition électronique

URL : http://journals.openedition.org/mappemonde/4438

DOI : $10.4000 /$ mappemonde.4438

ISSN : 1769-7298

Éditeur

UMR ESPACE

\section{Référence électronique}

Nicolas Bautès, « sylvain souchaud, Le Brésil », Mappemonde [En ligne], 129 | 2020, mis en ligne le 10 novembre 2020, consulté le 26 novembre 2020. URL : http://journals.openedition.org/mappemonde/ 4438 ; DOI : https://doi.org/10.4000/mappemonde.4438

Ce document a été généré automatiquement le 26 novembre 2020.

\section{(c) (i) (2)(2)}

La revue Mappemonde est mise à disposition selon les termes de la Licence Creative Commons Attribution - Pas d'Utilisation Commerciale - Partage dans les Mêmes Conditions 4.0 International. 


\section{SYLVAIN SOUCHAUD, Le Brésil}

Atlande, coll. « Clefs-concours »

Nicolas Bautès

\section{RÉFÉRENCE}

SYLVAin SOUCHAUd (2018). Le Brésil. Neuilly-sur-Seine : Atlande, coll. « Clefs concours-

Géographie ", $318 \mathrm{p}$. 
1 Deux ans après les faits relatés en introduction de l'ouvrage Le Brésil, écrit par Sylvain Souchaud, chercheur à l'IRD (URMIS), la situation politique du pays a continué à se complexifier et à se dégrader. La tendance observée par l'auteur, qui relatait l'escalade d'événements ayant profondément marqué l'ensemble de la société brésilienne, depuis la mise au jour fin 2014 d'un puissant système de corruption (dont l'affaire Lava Jato constitue le point d'orgue) jusqu'à l'empeachment (destitution) de la présidente Dilma Roussef en 2016, et l'emprisonnement en

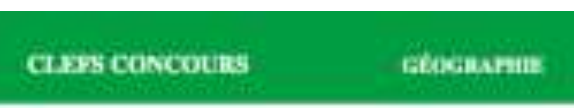

Sytraie Sowhoue

\section{Le Brésil}

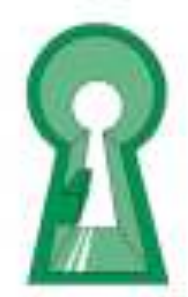

\section{Atronde} blanchiment d'argent, s'est fortement accélérée. Lors des élections de mai 2018, le vote brésilien a placé à la tête du pays le très controversé Jair Bolsonaro, dont les choix en matière économique autant que les valeurs morales et idéologiques annoncent des temps obscurs non seulement pour la démocratie, mais aussi pour l'environnement, les droits humains et particulièrement ceux des minorités et des travailleurs pauvres. Cette escalade tend à scinder plus encore qu'elle ne l'était la société brésilienne.

2 Face à une telle tension et à l'écho médiatique retentissant de chaque décision prise par ce gouvernement depuis son arrivée au pouvoir, il est difficile de ne pas « remarquer le désordre ", pour reprendre la formule empruntée au langage courant brésilien avec laquelle l'auteur ouvre son propos. C'est justement ce désordre, que l'optimisme brésilien a aujourd'hui tant de mal à dissimuler, que Sylvain Souchaud soumet à une fine analyse de ses expressions récentes et plus précisément encore de leurs logiques structurelles, au travers d'un objectif clairement affiché : « faire attention au désordre, lui donner un sens, comprendre ce qui se réalise et s'ordonne au cœur de la société brésilienne » (p. 13).

Tout au long des 7 chapitres qui composent cette courte synthèse, les traits saillants des espaces et des sociétés qui composent ce pays sont déployés sans pour autant que ne soit sacrifié le sens du particulier, qu'il soit événement historique, fait politique ou particularisme géographique. En 163 pages de texte, auxquelles s'ajoute un impressionnant nombre d'annexes ${ }^{1}$ que l'auteur rassemble dans une partie intitulée « outils » de près de 110 pages, il documente la géographie du Brésil en dressant une remarquable analyse des grands équilibres qui fabriquent ce pays, s'arrêtant sur ses principaux enjeux d'ordre social, politique et économique, revenant sur le processus historique de production du territoire en le mettant en perspective avec les puissants mouvements qui le traversent aujourd'hui.

L'armature générale qui soutient cette synthèse est posée dès les deux premiers chapitres de l'ouvrage. Y sont présentées tour à tour les «structures socio-spatiales » du pays (chap. 1), et sa dynamique régionale (chap. 2). 
5 Appuyée d'une série de données-clés et complétée de nombreuses cartes, schémas, tableaux et photographies (qui, malheureusement, sont en noir et blanc et consignés dans un cahier détaché du texte, ce qui permet, on le comprend, de limiter le prix de vente de l'ouvrage), cette analyse générale distille une première série de repères utiles. Déployés sur l'ensemble du texte, ils permettent de prendre la mesure (des superficies et des distances, des densités, des poids et des tendances, notamment économiques et démographiques...) sans pour autant obscurcir la compréhension générale des processus qui font le territoire. Au contraire, ces documents fournissent détails et vues synthétiques sur les dynamiques démographiques, sur les compositions sociale et ethnique de la population, les migrations, sur les tendances économiques, le travail et les structures foncières, l'auteur ne se limitant pas à inclure des données de seconde main sans en proposer des représentations cartographiques dont la lecture est particulièrement efficace.

6 C'est bien là l'un des intérêts majeurs de ce travail : à l'encyclopédisme de nombreux manuels de préparation aux concours, l'auteur oppose ici un ton juste et clair pour établir une ligne d'analyse forte et systématique: dynamiques de peuplement et modèle d'occupation (chap.1), construction de l'État et organisation du territoire (chap. 3), les processus historiques de peuplement sont analysés en lien étroit avec leurs logiques structurelles, depuis l'élaboration d'un modèle exportateur reposant sur l'exploitation conjointe des ressources du sol (sucre, or ou diamant, puis café et canne à sucre dans le littoral sud et son arrière-pays; caoutchouc et hévéa dans la région amazonienne) et des hommes (main-d'œuvre d'abord esclave puis "ouvrière »). Dynamique migratoire et logique pionnière sont ainsi analysées dans un même élan dès le chapitre 1, l'auteur revenant en quelques pages seulement sur cinq siècles d'occupation et de peuplement à propos desquels il interroge, en géographe, la part explicative du milieu et celle de l'histoire (la colonisation notamment) (p. 30).

7 Cette ligne d'analyse se prolonge pour gagner en détail et en nuance lorsque $\mathrm{S}$. Souchaud dresse le « portrait des cinq grandes régions brésiliennes» (p. 32), prenant la peine, au préalable, d'associer quelques caractères marquants pour mieux les présenter, caractères dont il précise qu'ils «ne leur sont pas exclusifs » et qu'il est possible de les retrouver un peu partout, plus ou moins affirmés (...) dans le reste du Brésil, à des degrés d'intensité divers : effet de répétition et de nuance qui donne forme à la mosaïque brésilienne » (p. 32).

8 Force de l'héritage colonial, persistance d'un modèle de développement économique largement dépendant de l'extérieur, composition ethnique de la population, mais aussi choix économiques, ces caractères se combinent de manières différenciées selon les régions. Afin d'expliquer et d'illustrer ces "répétitions et nuances", chaque grande région est abordée, dans ce chapitre 2 , à travers l'un de ses caractères majeurs.

9 L'expérience de la longue durée est celle que retient l'auteur pour décrire un Nordeste historiquement marginalisé, mais dont la dynamique de peuplement influe, au cours de différentes phases de migration, sur l'organisation de la population nationale. La récente «émergence » du pays, largement soutenue par les investissements fédéraux (notamment au moyen du Programme d'accélération de la croissance, PAC, lancé en 2007), laisse entrevoir une amélioration de sa production industrielle et une meilleure redistribution des richesses, même si le mal-développement demeure, traduit dans des indicateurs sociaux et démographiques toujours préoccupants, dans des inégalités sociales et infrarégionales fortes, et dans des niveaux de salaires généralement plus bas 
que dans les autres régions. Avec celle de l'Amazonie, la région Nordeste est aussi celle qui présente la situation de dégradation environnementale la plus préoccupante.

La région Sud-Est, quant à elle, est présentée comme le "cœur démographique et économique » du pays. S'y concentrent près de $92,9 \%$ de la population urbaine, principalement autour des trois pôles métropolitains de Sao Paulo, Rio de Janeiro et Belo Horizonte, et une part importante des investissements et de la production économique, dans un contexte démographique néanmoins préoccupant en raison d'un vieillissement accéléré de la population. Là encore, les principales étapes du développement de cette région depuis le XVIII ${ }^{e}$ siècle sont retracées, révélant le dispositif pionnier mis en place autour de l'économie des plantations, la structure sociale qu'elle contribue à établir sous l'effet d'un mouvement massif d'immigration et, progressivement, la transition de l'économie d'un modèle agraire et exportateur vers un modèle urbain et industriel (p. 41). Pour chacune de ces dimensions, l'auteur n'omet pas de signaler leurs effets sociaux et environnementaux.

11 "Centrale et convoitée ", tels sont les caractères associés à l'Amazonie, espace qui révèle nombre de paradoxes du pays et dont résonne à l'échelle internationale la vulnérabilité croissante, car, comme le souligne S. Souchaud, «l'internationalisation de la question amazonienne, qui débute dans les années 1980 (...) reste conflictuelle» (p. 49). Elle l'est d'autant plus dans le contexte politique actuel, marqué par les récentes mesures émanant du gouvernement comme celle de fusionner les ministères de l'Agriculture et de l'Environnement, laissant au secteur productif agro-industriel une large marge de manœuvre dans l'exploitation des ressources de cet espace. Sur chaque aspect de ces complexes thématiques, l'auteur ne manque pas là encore de renvoyer aux travaux de référence, particulièrement ceux de la géographie française.

Une même logique est proposée pour aborder les deux régions du Sud et du Centreouest, l'une au travers des expériences démocratiques conduites depuis plusieurs décennies, l'autre par son contraire, l'agronégoce. Entre ces deux régions en effet, les contextes politiques et les dynamiques socio-économiques semblent à bien des égards opposés.

13 Après avoir proposé quelques repères sur ces espaces, leurs dynamiques de peuplement et les enjeux socio-économiques auxquels ils font face dans la période récente, l'auteur décrit leurs principaux traits.

14 Le Sud est ainsi l'une des régions les plus avancées du point de vue démocratique, comme l'illustre le très médiatique budget municipal participatif mis en place à Porto Alegre au milieu des années 1980 ou, plus récemment, les très nombreuses initiatives observées tant dans le domaine économique et social que dans celui de l'urbanisme. Le dynamisme de l'agriculture familiale et du réseau des coopératives agricoles (près de 300 dans les seuls États du Paraná, de Santa Catarina et du Rio Grande do Sul, selon l'auteur) tend à faire de cette région l'une des plus stables et des plus avancées, souffrant de moins de déficits structurels que de nombreuses autres, en dépit de la récente montée de mouvements réactionnaires qui laissent entrevoir de nouveaux problèmes sociaux.

15 Le Centre-Ouest, quant à lui, semble au contraire souffrir de tendances lourdes largement liées à l'immensité de son territoire. Le déficit structurel en infrastructures, que l'espace et sa superficie n'expliquent pas à eux seuls, mais qui contribuent à complexifier les conditions de production économique, n'empêche pas néanmoins une économie hautement lucrative, fondée sur l'agriculture et l'élevage industriels, dont les 
propriétaires et investisseurs exercent une l'influence politique majeure à l'échelle nationale.

Cette approche régionale est ensuite prolongée par une série de chapitres thématiques, dont le chapitre 3, qui traite de la formation de l'État et présente le système politique brésilien en détail tout en replaçant le pays dans le monde, assure la transition.

Le chapitre suivant propose une fine étude démographique de cette population « en fin de transition ». La question migratoire occupe une place toute particulière dans cette analyse, abordée dans le cadre d'une réflexion sur la transition démographique caractéristique du pays, et sur les effets de mouvements historiques et contemporains de populations sur les dynamiques régionales (chapitre 4). On notera, dans ce même chapitre, la pertinence du «portrait d'une société en fin de transition démographique ", au cours duquel l'auteur s'attache, en dépit de la difficulté inhérente à cette entreprise, à « évaluer l'étendue » et « mesurer » les effets de cette transition. En effet, l'un des arguments forts de l'ouvrage est qu'elle a « une influence certaine sur la société brésilienne, car elle constitue en elle-même et indépendamment du contexte politique ou économique le cadre de circonstances historiques exceptionnelles, facteur de changements dont les gouvernements successifs des dernières décennies se sont plus ou moins saisis, que ce soit pour accompagner ces changements ou en tirer un bénéfice politique » (p. 87).

La coïncidence entre transition démographique et urbaine conduit l'auteur à faire état de la vigueur du processus démocratique observé dans le pays. L'éducation est une illustration majeure de cette tendance au changement, à la fois soutenue par des réformes politiques et stimulée par des aspirations sociales nouvelles. Les politiques mises en place en faveur des minorités expriment largement les avancées observées depuis deux décennies dans le domaine de l'éducation et, plus largement, dans un système de valeurs de plus en plus largement partagé par les Brésiliens. Reste que ce mouvement, fragile, demeure obstrué par un récent retournement de valeurs porté par la classe politique dirigeante, mais aussi par «la persistance de violentes inégalités sociales » (p. 91) et par une injustice structurelle, observées à toutes les échelles, à la fois entre les régions et au sein des espaces localisés. Ce constat mène l'auteur à souligner combien «les injustices criantes et systémiques (...) se déploient selon plusieurs modalités interdépendantes, tout à la fois spatiales, sociales et raciales, et constitutives du dualisme de la société brésilienne » (p. 93).

Révélateurs de ces inégalités et injustices, les désordres et violences en tous genres traversés par la société brésilienne font l'objet de développements au ton juste et pertinent, l'auteur prenant la peine d'en analyser les facteurs explicatifs : « insuffisance des investissements, manque de volonté politique, faible adhésion au modèle de l'Étatprovidence, discriminations structurelles ", expliquent la permanence d'un système (économique, social et politique) à deux-vitesses.

20 Ce portrait de la société brésilienne, concis et efficace, laisse place à trois chapitres qui viennent clore le propos. Cette géographie aborde tour à tour et de manière combinée les grandes problématiques et mutations du monde rural (chap.5), l'économie et les grands mouvements qui l'ont caractérisé depuis plus d'un demi-siècle (chap.6), pour enfin conclure sur le monde des villes et de la société urbaine (chap. 7).

21 Ce triptyque, pensé comme un tout, reprend et systématise les questions qui jalonnent le texte et qui définissent à la fois les traits saillants de l'espace brésilien et les défis auxquels il fait face. Le choix de traiter successivement du monde rural, de l'économie 
puis des villes permet à l'auteur de penser ensemble ces enjeux et de souligner leurs imbrications. Partant des structures sociales et économiques du pays, ancrées dans un monde rural en pleine mutation, l'auteur insiste sur l'origine des structures foncières et leurs dynamiques, fortement inégalitaires dès leur origine (p. 105), sur les tenants d'une économie agricole encore dominante et traversée de profonds conflits pour l'accès et le contrôle de la terre, avant de détailler les modalités selon lesquelles les pouvoirs successifs ont répondu aux problématiques de l'usage et de l'appropriation de la terre et de ses ressources. L'auteur oriente ensuite son propos sur les changements dans les conditions de travail et les modes de vie, tout en mettant l'accent sur les formes anciennes d'exploitation qui persistent dans une partie importante du pays (principalement dans le Nord et le Nordeste) et qui, en dépit d'un contexte économique favorable soutenu par des politiques publiques soucieuses de combler un déficit structurel en infrastructures, révèlent un espace où la brutalité de réalités sociales s'exprime par une pauvreté et une précarité persistantes.

Mais ce monde rural est aussi celui qui soutient l'activité exportatrice et le dynamisme de l'agriculture, "essence même du modèle colonial implanté par la Couronne portugaise » (p.111) et « vocation initiale (...) toujours active », fondé sur les ressources agricoles et sur l'élevage pour lesquels le Brésil est un producteur mondial de premier plan. Un rapide tour d'horizon des régions productives et de leurs ressources ouvre vers la question du modèle économique, " entre agrobusiness et agriculture familiale ", qui dissimule un univers social complexe (p.116) et profondément divisé. Alors qu'il propose un retour sur la trajectoire économique du pays dans la période récente, l'auteur s'interroge: émergence ou transition? Cette question, vivement débattue, figure au cœur du paradoxe brésilien. L'économie du pays, tout en ayant connu une croissance soutenue depuis les années 1990, a traversé une crise sévère dans les dernières années et demeure très dépendante des marchés internationaux. L'auteur rassemble des données récentes et variées relevant des domaines de l'économie et de la finance, de la consommation et de l'éducation, entre autres, pour souligner l'incertitude liée à la fin récente d'une " expérience de redistribution » (p. 137) mise en œuvre par les pouvoirs publics pendant plusieurs décennies, ce qui l'amène à penser, citant un récent travail de Kernstenetzky (2017), qu'elle «devrait accentuer les inégalités sociales et spatiales » (p. 137).

Le septième et dernier chapitre traite des villes et de la société urbaine. Il constitue en quelque sorte l'aboutissement de la réflexion géographique de l'auteur, pour laquelle il articule les nombreux phénomènes analysés tout au long de l'ouvrage et les replace dans le mouvement d'urbanisation du pays. L'accélération de la croissance urbaine observée depuis la fin du XIX siècle et l'établissement d'un réseau de villes à l'échelle nationale sont articulés à une réflexion synthétique sur l'histoire économique et démographique récente et ses singularités régionales.

L'auteur prolonge ensuite son propos sur les villes en opérant un changement d'échelle, apportant des éléments généraux sur la morphologie des centres-villes (qu'il nomme "région centre ») et leurs dynamiques récentes. Plus que de simples détails sur les structures matérielles de la ville, c'est une véritable géographie sociale urbaine qui est ici déployée, certes de manière partielle eu égard aux contraintes éditoriales, mais pertinente. Aux détails qui viennent illustrer et enrichir le propos, comme dans l'ensemble de l'ouvrage, l'auteur insiste sur les tensions qui marquent l'espace urbain brésilien (parmi d'autres, "la promotion immobilière soutenue par le marché 
financier », p. 158 ; « les habitants face aux acteurs économiques et à l'État » p. 159), qui le mène à conclure combien l'injustice spatiale est toujours à l'œuvre dans des villes intrinsèquement fragmentées.

À l'image de ces derniers développements, qui mêlent éléments d'analyse globaux et nuancés et illustrations issues d'études de cas localisées, cet ouvrage se présente comme une riche contribution non seulement à la compréhension de la géographie $d u$ Brésil, mais plus largement à celle des enjeux majeurs de notre temps. En dépit de leur nombre, de leur clarté et de leur diversité, les cartes, schémas, tableaux de données et photographies qui viennent compléter et clore le propos souffrent de leur isolement par rapport au texte, de leur taille souvent réduite, ce qui, on le comprend, résulte de contraintes éditoriales permettant notamment de limiter le prix de vente de l'ouvrage (19 euros). De telles contraintes de format associées à l'absence de couleur sont d'autant plus regrettables au vu de la pertinence de leur sélection et de la finesse de leur réalisation. Ces quelques détails n'affectent en rien la qualité de cette géographie régionale, dont l'analyse n'a rien à envier aux travaux théoriques et thématiques sur tous ces sujets tant elle s'attache les combiner, faisant de cet ouvrage un guide pour la compréhension du Brésil autant que pour celui de l'approche géographique.

\section{NOTES}

1. Cette partie se compose de deux courts textes proposant un zoom sur trois favelas de São Paulo et sur la récente colonisation agricole des milieux forestiers du sud-est du Roraima, suivi par une collection de 110 figures, 22 tableaux de données démographiques et statistiques, 38 cartes et croquis, 38 photos, une chronologie, un glossaire et une bibliographie de près de 300 références. 